\title{
Adjunct rasagiline to treat Parkinson's disease with motor fluctuations: a randomized, double-blind study in China
}

Zhenxin Zhang ${ }^{1^{*}} \mathbb{D}$, Ming Shao ${ }^{2}$, Shengdi Chen ${ }^{3}$, Chunfeng Liư ${ }^{4}$, Rong Peng ${ }^{5}$, Yansheng Li ${ }^{6}$, Jian Wang ${ }^{7}$, Suiqiang Zhu ${ }^{8}$, Qiumin Qu ${ }^{9}$, Xiaoying Zhang ${ }^{10}$, Haibo Chen ${ }^{11}$, Xiangru Sun ${ }^{12}$, Yanping Wang ${ }^{13}$, Shenggang Sun ${ }^{14}$, Baorong Zhang ${ }^{15}$, Jimei $\mathrm{Li}^{16}$, Xiaoping Pan ${ }^{17}$ and Gang Zhao ${ }^{18}$

\section{Abstract}

Background: The use of adjunct rasagiline in levodopa-treated patients with Parkinson's disease and motor fluctuations is supported by findings from large-scale clinical studies. This study is to investigate the efficacy and safety of adjunct rasagiline in Chinese patients with Parkinson's disease, as a product registration study.

Methods: This 16-week, randomized, double-blind, parallel-group, multicenter, placebo-controlled study of rasagiline $1 \mathrm{mg} /$ day included levodopa-treated patients with Parkinson's disease and motor fluctuations. The primary efficacy endpoint was mean change from baseline in total daily OFF time over 16 weeks. Secondary endpoints were Clinical Global Impressions - Improvement (CGI-I), and change in Unified Parkinson's Disease Rating Scale (UPDRS) Activities of daily living (ADL) and Motor scores. Patient well-being (EQ-5D), and the frequency of adverse events were also assessed.

Results: In total, 324 levodopa-treated patients were randomized to rasagiline $1 \mathrm{mg} /$ day $(n=165)$ or placebo $(n=159)$. Over 16 weeks, rasagiline statistically significantly reduced the mean [95\% confidence interval] total daily OFF time versus placebo $(-0.5 \mathrm{~h}[-0.92,-0.07] ; p=0.023)$. There were also statistically significant improvements versus placebo in CGI-I $(-0.4$ points $[-0.61,-0.22] ; p<0.001)$, UPDRS-ADL OFF $(-1.0$ points $[-1.75,-0.27] ; p=0.008)$, and UPDRS-Motor ON $(-1.6$ points $[-3.05,-0.14] ; p=0.032)$ scores, as well as the EQ-5D utility index $(p<0.05)$. Rasagiline was safe and well tolerated.

Conclusions: In levodopa-treated Chinese patients with Parkinson's disease and motor fluctuations, adjunct rasagiline $1 \mathrm{mg} /$ day statistically significantly reduced OFF time, and improved daily function and overall well-being, versus placebo. Consistent with findings in other countries, adjunct rasagiline was proven efficacious and well tolerated in Chinese patients.

Trial registration number: NCT01479530. Registered 22 November 2011.

Keywords: Parkinson's disease, Rasagiline, Adjunct, Motor fluctuations, OFF time, Quality of life, China

\footnotetext{
* Correspondence: wuzhangzhenxin@163.com

'Department of Neurology, Peking Union Medical College Hospital, 53

Dongdan N St, Dongcheng, Beijing, China

Full list of author information is available at the end of the article
}

(c) The Author(s). 2018 Open Access This article is distributed under the terms of the Creative Commons Attribution 4.0 International License (http://creativecommons.org/licenses/by/4.0/), which permits unrestricted use, distribution, and reproduction in any medium, provided you give appropriate credit to the original author(s) and the source, provide a link to the Creative Commons license, and indicate if changes were made. The Creative Commons Public Domain Dedication waiver (http://creativecommons.org/publicdomain/zero/1.0/) applies to the data made available in this article, unless otherwise stated. 


\section{Background}

\section{Prevalence of Parkinson's disease}

Parkinson's disease (PD) is a common, progressively disabling neurodegenerative disorder [1]. PD prevalence in China is estimated at $1.7 \%$ in individuals aged 65 or oldera rate comparable to that seen in European and other Asian countries [1, 2]. Moreover, the number of individuals over age 50 with $\mathrm{PD}$ in China is projected to rise to approximately 5 million by 2030 [3].

\section{Levodopa and motor fluctuations}

For decades, levodopa has been the mainstay of therapy for $\mathrm{PD}$ and it is considered effective in relieving the symptoms of disease [4]. Nevertheless, a major problem associated with levodopa treatment is the appearance of motor complications including fluctuations in mobility [5, 6] and drug-induced dyskinesia [7-9].

Motor fluctuations have been reported in more than half of patients receiving levodopa after 5 years of treatment [10]. A multicenter survey in China found the overall prevalence rate for wearing-off in Chinese PD patients to be $46.5 \%$, which is in line with reports from other countries [11]. Studies show that motor complications can appear relatively early, within a year of initiating levodopa $[5,12-14]$, and that the effectiveness of levodopa decreases with disease progression [6].

\section{Treatment with MAO-B inhibitors}

Therapies beyond levodopa have been developed with the aim of avoiding, or at least controlling, motor fluctuations (including wearing-off) and dyskinesia, while also facilitating symptom control. Administration of a monoamine oxidase type $\mathrm{B}$ (MAO-B) inhibitor aims to prolong the availability and activity of dopamine in the brain - slowing the elimination of endogenous dopamine, as well as exogenous dopamine derived from levodopa [15]. In terms of clinical outcome, MAO-B inhibitor therapy has been associated with improvements in motor fluctuations [16, 17] and, in the long-term, a reduced risk of dyskinesia in patients with PD [18]. Moreover, the Chinese Parkinson's Disease and Movement Disorder Society recommends MAO-B inhibitors for the management of wearing-off phenomena [19].

\section{Rasagiline}

Rasagiline is a potent MAO-B inhibitor [20]. Oral rasagiline has been approved for the treatment of idiopathic PD as monotherapy (without levodopa) or as adjunct therapy (with levodopa) in patients with end-of-dose fluctuations [21] in North America, Europe, and some Asian countries. The clinical efficacy, safety, and tolerability of rasagiline have been established in four Phase III studies (and one Phase II study) in predominantly Caucasian populations
$[16,17,22-24]$, and two Phase III studies in Chinese patients $[25,26]$. Meta-analyses have reinforced these findings [27-29]. The safety and tolerability of rasagiline in the Chinese population has also been demonstrated in a preliminary study of a transdermal patch formulation [30].

\section{Rationale for the current study}

To achieve product registration, the Chinese regulatory authorities require demonstration of efficacy and tolerability in a clinical trial conducted in the Chinese population [31]. The aim of the study was to evaluate the efficacy and safety of rasagiline (versus placebo) in levodopa-treated patients with PD and motor fluctuations, in a Chinese population.

Based on the submission, which included this study, rasagiline $\left(\right.$ Azilect $\left.^{\oplus}\right)$ received marketing authorization in China for the treatment of idiopathic PD as monotherapy (without levodopa) or as adjunct therapy (with levodopa) in patients with end of dose fluctuations (License No H20170336; 16 June 2017).

\section{Methods}

The study was a randomized, double-blind, parallel-group, placebo-controlled, multicenter trial conducted in China [Trial registration: NCT01479530].

Patients were enrolled at 18 study sites (neurologist clinics) between 20th December 2011 and 3rd June 2013. Each study site was granted approval by the respective hospital's ethics committee. Prior to enrolment, investigators provided patients with information about the study, and written, informed consent was obtained from all patients prior to any study-related activities.

The study was performed in compliance with the principles of Good Clinical Practice, and was designed and conducted in accordance with the principles of the Declaration of Helsinki [32].

\section{Patients}

The study enrolled male and female, adult ( $\geq 30$ years) Chinese outpatients with PD and motor fluctuations, who were receiving treatment with levodopa. Patients were selected from outpatient clinics, using competitive recruitment to encourage investigators to consider patients for the study (there was no fixed number per study site; rapid recruitment was encouraged; and study sites were remunerated on a per-patient basis).

Patient inclusion criteria were: diagnosis of idiopathic PD (at least two of resting tremor, bradykinesia, rigidity) without any other known or suspected cause of parkinsonism; optimized levodopa/dopa decarboxylase inhibitor (DDI) therapy, stable for 
$\geq 14$ days prior to baseline (3-7 doses per day, not including bedtime dose); and $\geq 1 \mathrm{~h} /$ day of OFF time (during waking hours). Other main inclusion criteria were: modified Hoehn and Yahr score $\leq 3$ in the ON state; stable dosing of any other anti-PD drug treatment for $\geq 30$ days prior to baseline, and throughout the study; and an ability to keep accurate 24-h patient diaries. Main exclusion criteria were: cognitive impairment (defined as a Mini Mental State Examination, MMSE, score $\leq 24$, i.e., at least mild cognitive impairment); melanoma or history of melanoma; previous neurosurgery for PD; presence of disabling dyskinesia; and known adverse reactions to tyramine-containing food. Disallowed concomitant medications included: MAO inhibitors (e.g., selegiline; within 90 days); antidepressants (within 42 days); sympathomimetics (including over-the-counter nasal or oral cold remedies); pethidine (within 14 days); and other psychotropic agents, including herbal (St John's Wort, ephedra - ma huang).

\section{Study procedure}

Informed consent was obtained during the screening visit. The screening period comprised 2 weeks of levodopa optimization (if required) and 2 weeks of stable levodopa treatment, followed by a 16-week doubleblind treatment period, and a 4-week safety follow-up after study treatment.

During the screening period, patients were trained in the use of the '24-h' diaries, after which they were required to be able to demonstrate ability to adequately complete three days of diaries before randomization took place. At baseline (start of the double-blind period), eligible patients were randomized (1:1) to receive either rasagiline $1 \mathrm{mg}$ (one tablet, once daily), or matching placebo, taken preferably in the morning, for 16 weeks. The dose of concomitant levodopa could be adjusted (decreased by omitting one dose/extending dosing interval; or increased back up to baseline dosage) during the first 4 weeks (if required), but remained constant thereafter. Randomization was achieved using computer-generated $\left(\mathrm{SAS}^{\oplus}\right.$, Version 9.2) randomization numbers from the study sponsor $(\mathrm{H}$. Lundbeck $\mathrm{A} / \mathrm{S})$, and a block (4) randomization method. Patients were randomized using an interactive voice/web response system (IV/WRS) hosted by Almac Clinical Technologies, Craigavon, United Kingdom. Study medication was supplied in wallet cards, with active and placebo treatment identical in appearance; the randomization code was not broken for any patient during the study. The study sponsor was responsible for study monitoring and other study-specific procedures.

\section{Treatment compliance}

At each visit after baseline, the patients were asked to return all the wallet cards, including unused investigational medicinal product (IMP). The investigator or his/ her designated staff were responsible for accounting for all IMP dispensed to and returned by the patients. The investigator asked patients who withdrew from the study for the date of their last dose of IMP.

\section{Assessments}

The primary efficacy endpoint was change from baseline in mean total daily OFF time, as assessed by patient home diaries [33] averaged over visits at Weeks 4, 8, 12 and 16. Patients were trained and assessed in their use of the diary. The patient diary was divided into 30-min intervals, from which the patient selected one of four options for each interval: asleep; OFF; ON with no dyskinesia or without troublesome dyskinesia; or $\mathrm{ON}$ with troublesome dyskinesia. Following a 20-to 30-min training session, the patient was allowed to complete the diary if, in the investigator's clinical opinion, the patient was able to assess their motor fluctuations correctly for a specified number of diary entries. During the screening visit, the patients completed $4 \mathrm{~h}$ (at least 8 diary ratings) of diary entries concurrently with, but independently from the investigator. There had to be at least $75 \%$ concordance in the diary entries between the patient and the investigator. The concordance had to include at least one ON to OFF, or OFF to ON, transition. The diary training session was to be repeated between the screening and baseline visits to achieve the $75 \%$ concordance requirement. For 3 days prior to each study visit, patients rated their status every $30 \mathrm{~min}$ as either 'asleep,' 'OFF,' 'ON with no dyskinesia/without troublesome dyskinesia' or 'ON with troublesome dyskinesia'. OFF time values were averaged over the 3 days, and $90 \%$ of entries had to be filled in correctly for the diary to be considered acceptable (i.e., no more than 5 missing entries per day).

Secondary efficacy endpoints were the Clinical Global Impressions - Improvement (CGI-I) [34] scale score during ON time at Week 16, change from baseline to Week 16 in the Unified Parkinson's Disease Rating Scale Activities of daily living (UPDRS-ADL) score [35] during OFF time, and UPDRS-Motor score during ON time.

Overall health assessments included the EuroQoL 5-dimension (EQ-5D) [36], and the Parkinson's Disease Questionnaire (PDQ-39) [37]. The scales used had been validated in the local language. The EQ-5D was assessed at baseline and at Weeks 2, 4, 8, 12 and 16; the PDQ-39 was assessed at baseline, Week 8 and Week 16 .

\section{Investigator and rater training}

The study investigators were trained in the requirements of Good Clinical Practice at the investigators' meetings 
or via an online system, and refresher training was provided. Rater training sessions were also conducted at the investigators' meetings or on site. The study investigators were to be adequately experienced with patients with PD to rate patients using the UPDRS and Modified Hoehn and Yahr Staging. Only neurologists approved by the sponsor's scales manager rated patients using the UPDRS and Modified Hoehn and Yahr Staging. MMSE was to be rated by a physician, and the CGI was rated by the investigator responsible for the patient. Only raters who qualified at a UPDRS rater qualification session were authorized to rate patients using the UPDRS.

Safety was assessed through the incidence of adverse events (AEs). Other safety endpoints included laboratory tests, vital signs, weight, electrocardiograms (ECGs), and physical and neurological examinations. Changes in UPDRS-Dyskinesia score (sum of items 32-34), and duration of 'ON time with troublesome dyskinesia' were also assessed in relation to safety.

\section{Statistical analysis}

The sample size calculation was based on the primary endpoint using a two-sided significance test at the 5\% level. Assuming a difference between rasagiline and placebo of $45 \mathrm{~min}$ in the mean change in total daily OFF time and a standard deviation (SD) of $2 \mathrm{~h}$, a sample size of 150 patients per treatment group gave a power of $90 \%$. Based on previous rasagiline studies, enrolment of 320 randomized patients was considered sufficient to secure 300 patients for the primary efficacy analysis.

Patient populations included the all-patients-randomized set (APRS), and all safety analyses were performed in the all-patients-treated set (APTS) of randomized patients who took at least one treatment dose. Efficacy was analyzed in the full-analysis set (FAS) of patients from the APTS who had at least one valid post-baseline measurement of total daily OFF time.

Primary and secondary endpoints were analyzed in a pre-planned hierarchical sequence: change in total daily OFF time (primary); CGI-I at Week 16; change from baseline in UPDRS-ADL OFF at Week 16; and change from baseline in UPDRS-Motor ON at Week 16. The testing continued from one endpoint to the next provided the difference from placebo was statistically significant in favor of rasagiline $(p<0.05)$ for that endpoint.

The primary efficacy endpoint was assessed using an analysis of covariance (ANCOVA) of the mean change from baseline in total daily OFF time (mean of changes over four study visits), with treatment and site as fixed factors and baseline total daily OFF time as a covariate, using observed cases (OC), as prespecified in the study protocol. Exploratory analyses included, change from baseline in mean total daily OFF time, analyzed for visits at Week $4,8,12$, and 16 by an ANCOVA model using last observation carried forward (LOCF) and OC principles, with treatment and center as fixed factors and baseline mean total daily OFF time as a covariate. A sensitivity analysis was also performed for the change from baseline in total daily OFF time using mixed model for repeated measures (MMRM), OC. This included a fixed effect of visit and site, baseline total daily OFF time as a covariate, treatment-by-visit interaction, and baseline-by-visit interaction, using an unstructured covariance matrix. If diaries were missing or unacceptable at a post-baseline visit, the total daily OFF time for that visit was calculated based on the remaining completed or acceptable diaries. An acceptable diary was defined as one with no more than 5 erroneous entries between 6 am and midnight. The same criterion was applied in the calculation of total daily $\mathrm{ON}$ time. If there were missing values at some visits (whole diaries not done or deemed unacceptable), the post-baseline average was calculated based on the remaining completed or acceptable diaries.

Secondary endpoints were analyzed by ANCOVA using LOCF with treatment and site as fixed factors and the corresponding baseline assessment as a covariate, with the exception of the analysis of CGI-I, which used the Clinical Global Impressions - Severity (CGI-S) score at baseline. Missing items at each visit for the UPDRS-ADL score during OFF time were replaced by the mean of the non-missing items, provided that the number of non-missing items was $\geq 10$; otherwise, the UPDRS-ADL score during OFF time for that visit was assigned a missing value. For the UPDRS-Motor score, the number of non-missing items had to be $\geq 20$. Missing values were imputed using the last observed value immediately prior to the missing value. Data from the withdrawal visit were assigned to a nominal visit using visit windows. If two competing assessments had the same nominal visit, then the originally observed visit was kept for by-visit analyses, whereas the withdrawal visit was used for the LOCF analyses.

EQ-5D and PDQ-39 scores were analyzed by ANCOVA (OC), with treatment and site as fixed factors and the respective baseline score as a covariate. Missing items for the EQ-5D were not imputed. For the PDQ-39 dimensions, missing data were integrated in the scoring algorithm using the following rule: a dimension score was calculated only if $\geq 50 \%$ of its constitutive items had valid answers.

No interim analyses were planned.

Statistical analyses were conducted by $\mathrm{H}$. Lundbeck A/S. The principal statistical software used was SAS ${ }^{\oplus}$, Version 9.2.

\section{Results}

\section{Baseline characteristics}

A total of 366 patients were screened, and 324 patients were randomized - as shown in Fig. 1 - and the overall 


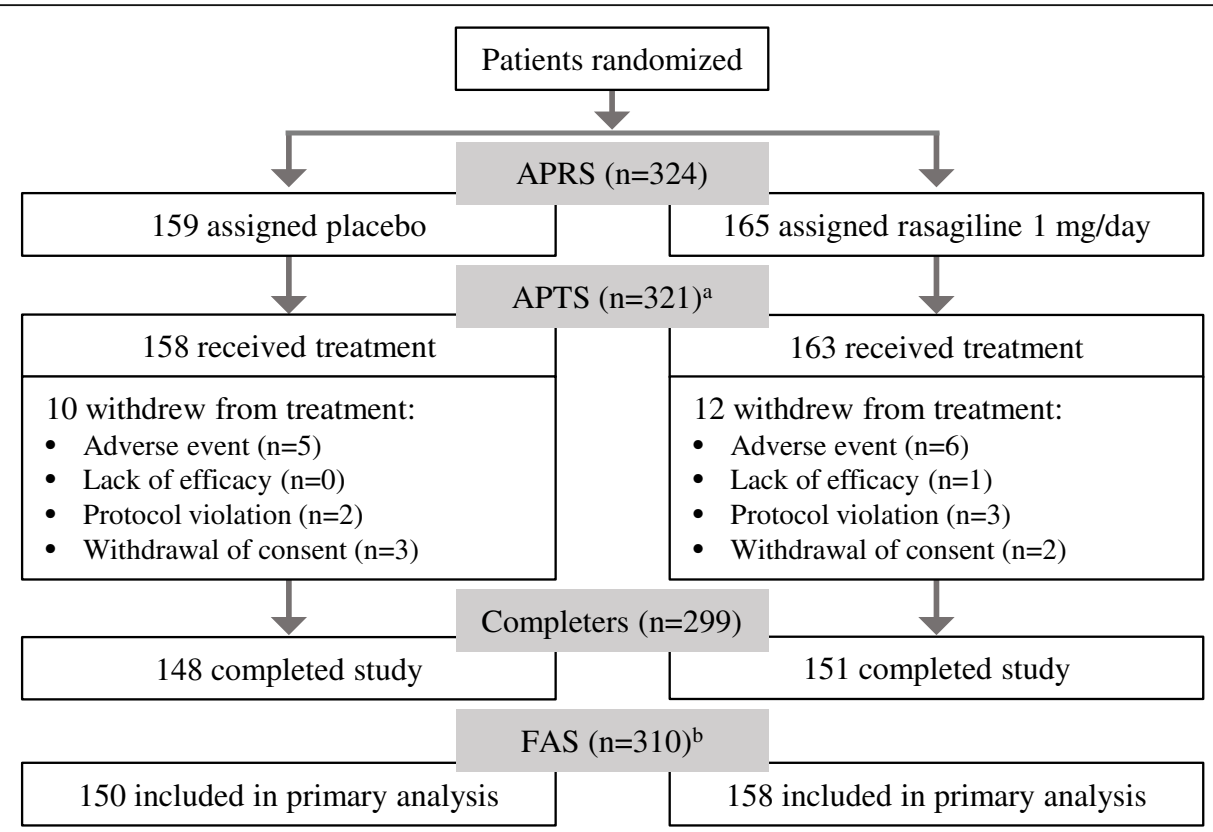

Fig. 1 Flow of patients through the study. ${ }^{3} 3$ patients did not receive treatment and were excluded from the APTS. ${ }^{b} 11$ patients did not have valid post-baseline diaries and were excluded from the FAS; 2 further patients (in the placebo group) were excluded from the primary efficacy analysis due to having no baseline diaries, but were in the FAS for all non-diary-related endpoints. APRS = all-patients-randomized set; APTS = all-patients-treated set; FAS = full-analysis set (modified intent-to-treat)

withdrawal rate was low (22 patients; 7\%). Patient demographics and clinical characteristics were similar between the two treatment groups at baseline (Table 1). The population was Asian (Chinese), with a mean age of 62 years (range 32-83 years), mean disease duration of approximately 7 years, and mean daily OFF time of $6.1 \mathrm{~h}$.

\section{Patient diaries and compliance with IMP Patient diaries}

A total of 174,816 post-baseline diary records were assessed, of which $<1 \%$ were considered unacceptable -placebo $0.4 \%$ (387/86,256); rasagiline $0.6 \%(532 / 88,560)$.

\section{Compliance with IMP}

The mean number of days of exposure to IMP was approximately 107 days in the placebo group and approximately 106 days in the rasagiline group. The majority of the patients received IMP for between 85 and 112 days (68\% in the placebo and $76 \%$ in the rasagiline group). The mean compliance with IMP was $\geq 99 \%$ (placebo group: 86-100\%; rasagiline group: $88-100 \%$ ).

\section{Efficacy analyses}

The FAS consisted of 310 patients, two of whom were excluded from all analyses of OFF and ON time (including primary analysis) as they had no diary entries at baseline.

\section{Change in Total daily OFF time}

In the primary efficacy analysis, the mean change from baseline in total daily OFF time was statistically significantly greater in the rasagiline-treated group than in the placebo group, with a treatment difference of $-0.5 \mathrm{~h}$, in favor of rasagiline (Fig. 2a, Table 2). Results of the exploratory analyses of mean change from baseline in total daily OFF time showed similar results to the primary efficacy analysis (Table 2). The ANCOVA OC and the MMRM sensitivity analysis were consistently in favor of rasagiline at all timepoints, although this reached statistical significance at Week 4 only in both analyses. Results of the ANCOVA, LOCF analysis were consistently in favor of rasagiline at all timepoints, and reached statistical significance at Weeks 4 and 16 (Fig. 2b, Table 2).

\section{Motor symptoms, daily function, and global outcomes}

Tested in the defined hierarchical testing sequence, the primary and secondary endpoints were all statistically significantly in favor of rasagiline versus placebo (Table 2). Findings indicated that rasagiline produced statistically significant benefits versus placebo in overall global improvement (CGI-I), daily function during OFF time (UPDRS-ADL OFF), and motor symptoms during ON time (UPDRS-Motor ON) at Week 16.

\section{Health-related quality of life}

Rasagiline produced a statistically significant improvement versus placebo in the PDQ-39 dimensions of activities of 
Table 1 Patient demographics and characteristics at baseline (all-patients-treated set)

\begin{tabular}{|c|c|c|}
\hline & Placebo $(n=158)$ & Rasagiline $1 \mathrm{mg} /$ day $(n=163)$ \\
\hline Age, years & $61.7(9.9)$ & $62.7(8.9)$ \\
\hline Gender, male & 109 (69\%) & $103(63 \%)$ \\
\hline $\mathrm{BMl}$ & $23.2(3.3)$ & $23.1(3.2)$ \\
\hline \multicolumn{3}{|l|}{ Education (highest level) ${ }^{a}$} \\
\hline Elementary/middle school & $67(44 \%)$ & $68(43 \%)$ \\
\hline High school & $45(30 \%)$ & $41(26 \%)$ \\
\hline College/university/graduate & $33(22 \%)$ & $46(29 \%)$ \\
\hline None/other & $7(5 \%)$ & $3(2 \%)$ \\
\hline Duration of $\mathrm{PD}$, years & $7.1(4.3)$ & $7.4(4.8)$ \\
\hline Modified Hoehn and Yahr ON & $2.0(0.7)$ & $1.9(0.7)$ \\
\hline Patients with dyskinesia & $31(20 \%)$ & 49 (30\%) \\
\hline UPDRS-Dyskinesia & $0.35(0.9)$ & $0.49(1.2)$ \\
\hline ON time with troublesome dyskinesia, hours & $0.55(1.8)$ & $0.67(2.0)$ \\
\hline \multicolumn{3}{|l|}{ Patients taking concomitant anti-PD medications: ${ }^{\text {b }}$} \\
\hline Anticholinergics & $20(13 \%)$ & $21(13 \%)$ \\
\hline Amantadine & $58(37 \%)$ & $50(31 \%)$ \\
\hline Dopamine agonists ${ }^{c}$ & $102(65 \%)$ & $104(64 \%)$ \\
\hline COMT inhibitors $^{d}$ & $31(20 \%)$ & $34(21 \%)$ \\
\hline Data for FAS population & $(n=152)$ & $(n=158)$ \\
\hline Total daily OFF time, hours ${ }^{\mathrm{e}}$ & $6.1(2.7)$ & $6.1(2.6)$ \\
\hline Total daily ON time, hours ${ }^{\mathrm{e}}$ & $9.3(2.5)$ & $9.4(2.4)$ \\
\hline UPDRS-ADL OFF & $16.5(7.5)$ & $15.6(7.2)$ \\
\hline UPDRS-ADL ON & $7.3(5.1)$ & $6.8(4.6)$ \\
\hline UPDRS-Motor ON & $25.6(10.5)$ & $23.8(10.5)$ \\
\hline CGI-S & $4.1(0.8)$ & $3.9(0.8)$ \\
\hline Total daily levodopa dose, mg & $550(224)$ & $501(222)$ \\
\hline Total daily levodopa dose at end of Week $4, \mathrm{mg}^{\mathrm{f}}$ & $549(225)$ & 495 (219) \\
\hline
\end{tabular}

${ }^{\mathrm{a}} \mathrm{FAS}$ population ( $n=152$ for placebo group, $n=158$ for rasagiline group)

${ }^{\mathrm{b}}$ medication that was continued after the first study treatment dose

cbromocriptine, piribedil, pramipexole, pramipexole dihydrochloride; ropinirole hydrochloride

dentacapone

$e_{n}=150$ for placebo group

${ }^{f}$ FAS, OC ( $n=151$ for placebo group, $n=157$ for rasagiline group)

daily living $(-6.1 \pm 1.8$ [standard error] points; $p<0.001)$ and bodily discomfort $(-3.9 \pm 1.9$ points; $p=0.043)$ (Table 2). The patients' general well-being (EQ-5D utility index; $0.05 \pm 0.02$ points; $p=0.024$ ) and perception of their own health state (EQ-5D visual analogue scale, VAS; $4.31 \pm 1.59$ points; $p=0.007$ ) were also statistically significantly improved with rasagiline treatment versus placebo (Table 2).

\section{Safety analyses}

The overall incidence of AEs was similar between the rasagiline (40.5\%) and placebo (37.3\%) groups (Table 3), with only one treatment-emergent adverse event (TEAE) occurring at a frequency of $\geq 5 \%$ in either group: dyskinesia was reported in $7.6 \%$ of placebo-treated patients and
$6.7 \%$ of rasagiline-treated patients. The majority of TEAEs were mild or moderate, in both treatment groups. The rate of withdrawal due to AEs was low and similar between the rasagiline (3.7\%) and placebo (3.2\%) groups, as was the incidence of serious adverse events (SAEs) (4.3\% for rasagiline and 3.2\% for placebo) (Table 3). No deaths occurred during the study.

There were no clinically relevant changes in clinical safety laboratory values, vital signs, weight, or ECG status. The mean (SD) change in UPDRS-Dyskinesia score was slight: placebo, 0.05 (0.59) points; rasagiline, $0.13(0.94)$ points. The mean duration of $\mathrm{ON}$ time with troublesome dyskinesia remained relatively unchanged from baseline to Week 16 in the placebo $(0.55$ and $0.65 \mathrm{~h}$, respectively) and rasagiline groups ( 0.67 and $0.87 \mathrm{~h}$, respectively). 
a

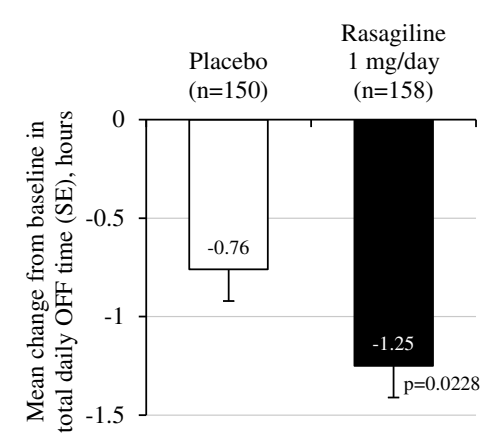

b

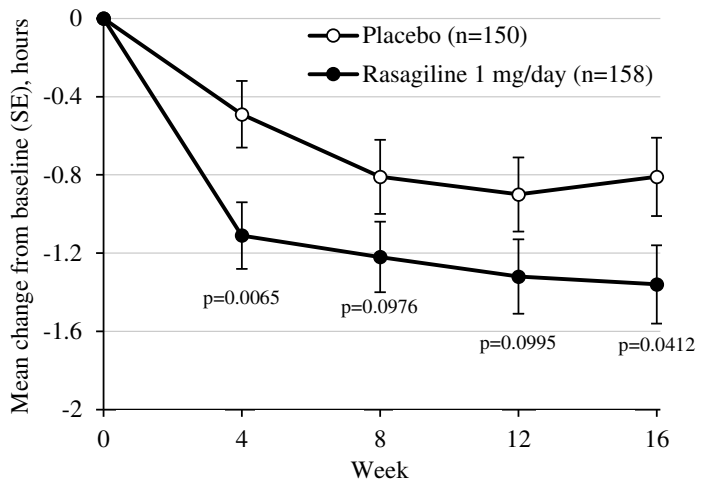

Fig. 2 Changes from baseline in total daily OFF time. a Primary endpoint: mean change from baseline, averaged over visits at Weeks 4, 8, 12 and 16, in total daily OFF time (full-analysis set, analysis of covariance, observed cases). b Adjusted change from baseline in total daily OFF time (full-analysis set, analysis of covariance, last observation carried forward). SE = standard error

Table 2 Change from baseline in efficacy endpoints (full-analysis set)

\begin{tabular}{|c|c|c|c|c|}
\hline & \multicolumn{2}{|c|}{ Adjusted mean change from baseline (SE) } & \multirow{2}{*}{$\begin{array}{l}\text { Difference vs } \\
\text { placebo ( } 95 \% \text { Cl) }\end{array}$} & \multirow[t]{2}{*}{$p$-values } \\
\hline & Placebo $(n=152)$ & Rasagiline $1 \mathrm{mg} /$ day $(n=158)$ & & \\
\hline \multicolumn{5}{|c|}{ Primary endpoint (mean change over Weeks 4, 8, 12, and 16; ANCOVA, OC) } \\
\hline Total daily OFF time, hours ${ }^{a}$ & $-0.76(0.16)$ & $-1.25(0.16)$ & $-0.50(-0.92,-0.07)$ & 0.023 \\
\hline \multicolumn{5}{|c|}{ Secondary endpoints (Week 16; ANCOVA, LOCF) } \\
\hline$C G I-I^{b}$ & $3.56(0.07)$ & $3.15(0.07)$ & $-0.41(-0.61,-0.22)$ & $<0.001$ \\
\hline UPDRS-ADL OFF & $-1.20(0.28)$ & $-2.21(0.28)$ & $-1.01(-1.75,-0.27)$ & 0.008 \\
\hline UPDRS-Motor ON & $-1.75(0.55)$ & $-3.34(0.55)$ & $-1.60(-3.05,-0.14)$ & 0.032 \\
\hline \multicolumn{5}{|c|}{ Exploratory analyses (mean change from baseline to Week 16 in total daily OFF time) } \\
\hline ANCOVA, OC & $-0.87(0.21)(n=147)$ & $-1.39(0.21)(n=151)$ & $-0.52(-1.06,0.02)$ & 0.0602 \\
\hline ANCOVA, LOCF & $-0.81(0.20)$ & $-1.36(0.20)$ & $-0.55(-1.08,-0.02)$ & 0.0412 \\
\hline MMRM, OC & $-0.81(0.20)$ & $-1.34(0.20)$ & $-0.53(-1.07,0.00)$ & 0.0514 \\
\hline \multicolumn{5}{|c|}{ PDQ-39 summary index and dimension scores (mean change, SE at Week 16; ANCOVA, OC) } \\
\hline Summary index & $-0.1(0.8)$ & $-1.9(0.8)$ & $-1.8(-3.96,0.42)$ & 0.1122 \\
\hline Activities of daily living & $2.0(1.3)$ & $-4.0(1.3)$ & $-6.1(-9.52,-2.64)$ & $<0.001$ \\
\hline Bodily discomfort & $1.7(1.4)$ & $-2.2(1.4)$ & $-3.9(-7.65,-0.12)$ & 0.043 \\
\hline Cognition & $0.9(1.2)$ & $0.4(1.2)$ & $-0.6(-3.77,2.67)$ & 0.737 \\
\hline Communication & $-1.0(1.2)$ & $0.1(1.2)$ & $1.1(-2.09,4.23)$ & 0.506 \\
\hline Emotional well-being & $-0.6(1.5)$ & $-3.0(1.5)$ & $-2.4(-6.35,1.49)$ & 0.224 \\
\hline Mobility & $-1.6(1.2)$ & $-4.1(1.2)$ & $-2.5(-5.59,0.53)$ & 0.104 \\
\hline Social support & $-1.2(1.1)$ & $0.4(1.1)$ & $1.6(-1.35,4.53)$ & 0.288 \\
\hline Stigma & $-2.2(1.4)$ & $-4.7(1.4)$ & $-2.5(-6.22,1.32)$ & 0.202 \\
\hline \multicolumn{5}{|c|}{ EQ-5D (mean change, SE at Week 16; ANCOVA, OC) } \\
\hline Utility index & $0.00(0.02)$ & $0.05(0.02)$ & $0.05(0.01,0.09)$ & 0.024 \\
\hline Health state ${ }^{c}$ & $0.77(1.18)$ & $5.09(1.20)$ & $4.31(1.18,7.45)$ & 0.007 \\
\hline
\end{tabular}

${ }^{a} n=150$ for placebo group

babsolute value at Week 16

cmeasured using visual analogue scale 
Table 3 Frequency of adverse events (all-patients-treated set)

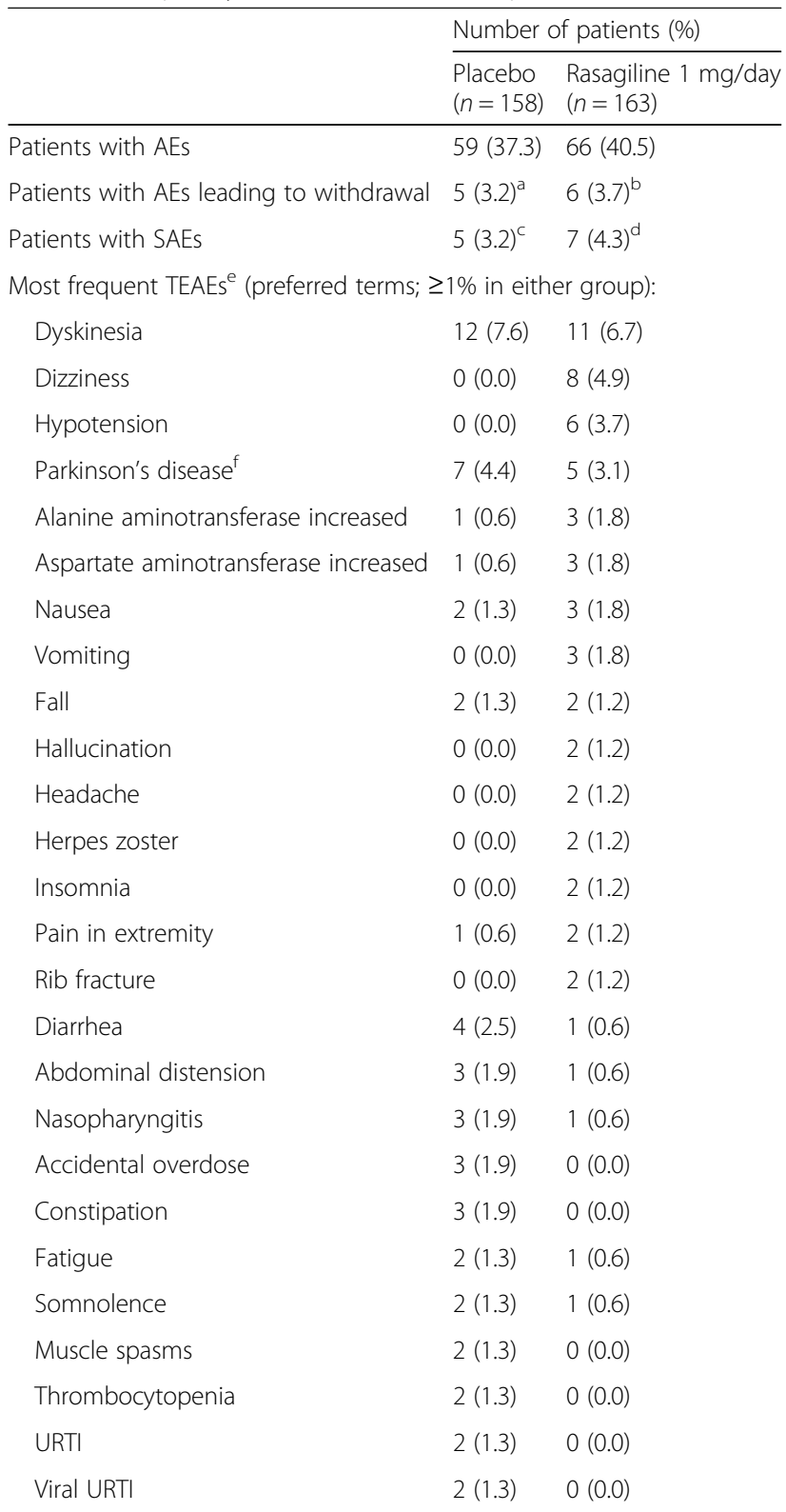

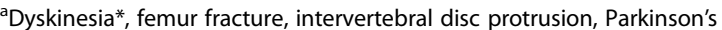
disease*, psoriasis* (all $n=1$ )

${ }^{b}$ hallucination* $(n=2)$, dyskinesia*, epilepsy, hypotension*, transient ischemic attack (all $n=1$ )

'edema peripheral, erysipelas* + psoriasis*, femur fracture, intervertebral disc protrusion, multiple fractures + road traffic accident (all $n=1$

dappendicitis, delusional perception*, Parkinson's disease*, peripheral nerve injury, sick sinus syndrome, transient ischemic attack, venous stenosis (all $n=1$ )

ean event that started after the first dose of study treatment and prior to the last protocol-specified contact with that patient

f patients reported symptom aggravation/disease progression

"Considered by the investigator to be possibly or probably related to treatment

\section{Discussion}

Building on the findings of two large-scale registration studies (LARGO and PRESTO) set in Europe/America $[16,17]$, the current study investigated the efficacy and safety of rasagiline $1 \mathrm{mg} /$ day as adjunct to levodopa in a Chinese population of patients with PD and motor fluctuations. Although rasagiline had been extensively studied, with proven efficacy and tolerability [38], for product registration purposes the Chinese regulatory authorities require a further study in a Chinese population to support marketing authorization in China. Thus, the study reported here was undertaken to support the marketing authorization submission for rasagiline as adjunct treatment (with levodopa) for patients with idiopathic PD and end-ofdose fluctuations - and adds to the overall clinical evidence base for rasagiline.

The primary efficacy analysis of reduction in mean total daily OFF time revealed a statistically significant difference of $30 \mathrm{~min}$ between the rasagiline and placebo groups. The effect of rasagiline in reducing daily OFF time $(-1.25 \mathrm{~h})$ was comparable to that seen in Caucasian patients in the LARGO study $(-1.18 \mathrm{~h})$, although the placebo response in the current study was greater than in LARGO ( $-0.76 \mathrm{~h}$ compared with $-0.40 \mathrm{~h}$ ) [16]. The placebo response seen here was also greater than that seen in a similar study of rasagiline in Chinese patients $(-0.69 \mathrm{~h})$ although, in that study, there was a greater reduction in OFF time $(-1.75 \mathrm{~h})$ associated with rasagiline treatment [25]. The occurrence of a placebo response in randomized clinical studies is well documented, prominent in PD [39], and has been observed in both Caucasian and Asian populations. Placebo response in PD has been linked with an increased endogenous dopamine release (and resulting symptom alleviation), possibly related to a good doctor-patient relationship, and the patient's expectation of clinical benefit (involving reward learning enhancement and the appraisal network) [39-42]. A South Korean study of adjunct entacapone in patients with wearing-off phenomena, reported a placebo response of approximately a 1 -h reduction in daily OFF time [43] - a magnitude of effect comparable to that observed in our study.

In a 12-week study of PD patients with motor fluctuations, conducted in various geographical regions at approximately the same time as our study in Chinese PD patients, rasagiline $1 \mathrm{mg}$ /day (included as active comparator), despite reducing daily OFF time by more than $1 \mathrm{~h}$, failed to demonstrate statistically significant separation from placebo in total daily OFF time (rasagiline $-1.1 \mathrm{~h}$, placebo $-0.8 \mathrm{~h}, p=0.28$ ). [44] The observed large placebo response was considered to be a factor contributing to the failure of the study, as were various study design and conduct issues. [44] Regardless of patient ethnicity, the results of our study presented here serve to reconfirm the efficacy of rasagiline as adjunctive therapy in patients with PD experiencing motor fluctuations.

Concurrent with the reduction in OFF time, statistically significant improvements in the secondary efficacy endpoints (CGI-I score, UPDRS-ADL score during OFF time, 
and UPDRS-Motor score during ON time) were also observed with rasagiline, which supported the results of the primary efficacy analysis.

Results of the primary and secondary analyses also correlated with meaningful improvements in overall health and the patient's perception of their own well-being. The improvements in PDQ-39 scores in the rasagiline group indicated that these patients had a slightly better quality of life than those in the placebo group, with statistically significant benefits in the dimensions of ADLs and bodily discomfort.

It is notable that there was a lower UPDRS-Dyskinesia score (0.35-0.49, i.e., less severe symptoms) at baseline in this Chinese study group, compared to that recorded in the LARGO study (1.4-1.5) [16]. This could result from the much lower daily dosage of levodopa (501$550 \mathrm{mg}$ ) in the Chinese study population compared to that of the LARGO study (697-722 mg), since the severity of disease, and concomitant use of anti-PD medications, at baseline are comparable between the two study populations [16]. Lower dose of levodopa at baseline (515-521 mg) was also seen in the Zhang et al. (2013) study of rasagiline in Chinese patients [25]. Additionally, a large cross-sectional survey in four urban regions of China revealed that many Chinese PD patients are treated with low-dose levodopa - medical practice that might be influenced by Chinese culture [45].

It was also observed that the improvements in motor symptoms/control (related to an improved supply of dopamine) were not accompanied by a worsening of dyskinesia in this Chinese study. At baseline, more patients in the rasagiline group suffered from dyskinesia compared with the placebo group. However, during the study the reporting of dyskinesia as a TEAE was similar between the placebo (7.6\%) and rasagiline (6.7\%) groups, and there were only small changes in UPDRS-Dyskinesia score, and in the duration of 'ON time with troublesome dyskinesia' (patient diaries), from baseline to Week 16. These results were consistent with a cohort study, which showed that MAO-B inhibitor therapy was associated with reduced risk of dyskinesia in patients with PD [18].

Overall, treatment with rasagiline was safe and well tolerated, with AEs reported at similar levels to the placebo group, and no new safety concerns observed. Indeed, safety findings were comparable to those of the (predominantly European) LARGO study [16] in terms of the incidence of dyskinesia, and the low incidence of withdrawals due to AEs. The findings are also consistent with existing study data demonstrating that the pharmacokinetics (and associated tolerability) of rasagiline were similar in Chinese and Caucasian populations [46].

Given that patients with PD require long-term therapy, a potential limitation of this study is the short duration of treatment (4 months). The effect of rasagiline in Chinese PD patients could be examined further in a long-term trial.

\section{Conclusions}

In conclusion, rasagiline statistically significantly reduced OFF time in Chinese patients with PD and motor fluctuations. Despite the impact of a larger than expected placebo response, the 30 -min reduction in disabling OFF time per day in the rasagiline group is notable. In addition, improvements were observed in the secondary and quality of life/general well-being endpoints in the rasagiline group, which support the primary endpoint. Consistent with data from similarly robust, well-controlled studies in other world locations, data from this Chinese study show that rasagiline is well tolerated, and a clinically useful adjunct therapy to levodopa in patients with PD.

\section{Abbreviations \\ ADL: Activity of daily living; AE: Adverse event; ANCOVA: Analysis of covariance; APRS: All-patients-randomized set; APTS: All-patients-treated set; BMI: Body mass index; CGI-I: Clinical Global Impressions - Improvement; CGI-S: Clinical Global Impressions - Severity; Cl: Confidence interval; COMT: Catechol-O-methyltransferase; DDI: Dopa decarboxylase inhibitor; ECG: Electrocardiogram; EQ-5D: EuroQoL 5-dimension; FAS: Full-analysis set; IMP: Investigational medicinal product; IV/WRS: Interactive voice/web response system; LOCF: Last observation carried forward; MAO- B: Monoamine oxidase type B; MMRM: Mixed model for repeated measures; MMSE: Mini Mental State Examination; OC: Observed cases; PD: Parkinson's disease; PDQ-39: 39-item Parkinson's Disease Questionnaire; SAE: Serious adverse event; SD: Standard deviation; SE: Standard error; \\ TEAE: Treatment-emergent adverse event; UPDRS: Unified Parkinson's Disease Rating Scale; URTI: Upper respiratory tract infection}

\section{Acknowledgements}

Writing support was provided by Cambridge Medical Communication Ltd. (Cambridge, UK).

The authors thank the participating subjects and staff, and the investigators for their contributions to the study. The primary investigators for this study were Zhenxin Zhang, Ming Shao, Baorong Zhang, Xiaoping Pan, Chunfeng Liu, Qiang Dong, Xiaoying Zhang, Shenggang Sun, Gang Zhao, Dong Zhou, Shengdi Chen, Yansheng Li, Qiumin Qu, Xiangru Sun, Jimei Li, Haibo Chen, Suiqiang Zhu, and Yanping Wang.

\section{Funding}

The study was sponsored by H. Lundbeck A/S, Copenhagen, Denmark. Writing support was funded by $H$. Lundbeck $A / S$.

\section{Availability of data and materials}

The data that support the findings of this study are available from $\mathrm{H}$. Lundbeck A/S, Copenhagen, Denmark but restrictions apply to the availability of these data, which were used under license for the current study, and so are not publicly available. Data are, however, available from the authors upon reasonable request and with permission of $\mathrm{H}$. Lundbeck A/S, Copenhagen, Denmark.

\section{Authors' contributions}

All authors contributed to the design of the study, and interpretation of data, and read and approved the final manuscript. ZZ: Study concept and design, data review/comments, interpretation of data, study supervision, manuscript preparation (review and critique). MS, SC, CL, RP, YL, JW, SZ, QQ, $X Z, H C, X S, Y W, S S, B Z, J L, X P, G Z$ : Study design and organization, execution, and manuscript review/critique.

\section{Ethics approval and consent to participate}

Each study site was granted approval by the respective hospital's ethics committee. Prior to enrolment, investigators provided patients with information about the study, and written, informed consent was obtained 
from all patients prior to any study-related activities. The study was performed in compliance with the principles of Good Clinical Practice, and was designed and conducted in accordance with the principles of the Declaration of Helsinki.

\section{Consent for publication}

Not applicable.

\section{Competing interests}

The authors declare that they have no competing interests.

\section{Author details}

'Department of Neurology, Peking Union Medical College Hospital, 53 Dongdan N St, Dongcheng, Beijing, China. ${ }^{2}$ Department of Neurology, The First Affiliated Hospital of Guangzhou Medical University, Guangzhou, China. ${ }^{3}$ Department of Neurology, Ruijin Hospital, Shanghai Jiaotong University School of Medicine, Shanghai, China. ${ }^{4}$ Department of Neurology, The Second Affiliated Hospital of Soochow University, Suzhou, China. ${ }^{5}$ Department of Neurology, West China Hospital, Sichuan University, Chengdu, China. ${ }^{6}$ Department of Neurology, Renji Hospital, Shanghai Jiaotong University School of Medicine, Shanghai, China. ${ }^{7}$ Department of Neurology, Huashan Hospital, Fudan University, Shanghai, China. ${ }^{8}$ Department of Neurology, Tongji Hospital of Tongji Medical College, Huazhong University of Science and Technology, Wuhan, China. ${ }^{9}$ Department of Neurology, The First Affiliated Hospital of Xi'an Jiaotong University, Xi'an, China. ${ }^{10}$ Department of Neurology, Beijing Tiantan Hospital, Capital Medical University, Beijing, China. ${ }^{11}$ Department of Neurology, Beijing Hospital, Beijing, China. ${ }^{12}$ Department of Neurology, Peking University First Hospital, Beijing, China. ${ }^{13}$ Department of Neurology, The Second Affiliated Hospital of Guangzhou Medical University, Guangzhou, China. ${ }^{14}$ Department of Neurology, Union Hospital, Tongji Medical College, Huazhong University of Science and Technology, Wuhan, China. ${ }^{15}$ Department of Neurology, The Second Affiliated hospital of Zhejiang University School of Medicine, Zhejiang, Hangzhou, China. ${ }^{16}$ Department of Neurology, Beijing Friendship Hospital, Capital Medical University, Beijing, China. ${ }^{17}$ Department of Neurology, Guangzhou First People's Hospital, Guangzhou, China. ${ }^{18}$ Department of Neurology, Xijing Hospital, The First Affiliated Hospital of The Fourth Military Medical University, Xi'an, China.

\section{Received: 19 May 2018 Accepted: 19 June 2018}

Published online: 30 June 2018

\section{References}

1. Ma CL, Su L, Xie JJ, Long JX, Wu P, Gu L. The prevalence and incidence of Parkinson's disease in China: a systematic review and meta-analysis. J Neural Transm (Vienna). 2014;121:123-34.

2. Zou YM, Liu J, Tian ZY, Lu D, Zhou YY. Systematic review of the prevalence and incidence of Parkinson's disease in the People's republic of China. Neuropsychiatr Dis Treat. 2015;11:1467-72.

3. Dorsey ER, Constantinescu R, Thompson JP, Biglan KM, Holloway RG, Kieburtz K, et al. Projected number of people with Parkinson disease in the most populous nations, 2005 through 2030. Neurology. 2007;68:384-6.

4. Paulson GW. Management of the patient with newly-diagnosed Parkinson's disease. Geriatrics. 1993:48(30-4):39-40.

5. Shaw KM, Lees AJ, Stern GM. The impact of treatment with levodopa on Parkinson's disease. Q J Med. 1980;49:283-93.

6. Marsden CD, Parkes JD. Success and problems of long-term levodopa therapy in Parkinson's disease. Lancet. 1977;1:345-9.

7. Stocchi F, Jenner P, Obeso JA. When do levodopa motor fluctuations first appear in Parkinson's disease. Eur Neurol. 2010;63:257-66.

8. Marsden CD, Parkes JD. "On-off" effects in patients with Parkinson's disease on chronic levodopa therapy. Lancet. 1976;1:292-6.

9. Marsden CD, Parkes JD, Quinn N. Fluctuations of disability in Parkinson's disease - clinical aspects. In: Marsden CD, Fahn S, editors. Movement disorders. London: Butterworth Scientific; 1982. p. 96-122.

10. Sweet RD, McDowell FH. Five years' treatment of Parkinson's disease with levodopa. Therapeutic results and survival of 100 patients. Ann Intern Med. 1975;83:456-63.

11. Chen W, Xiao Q, Shao M, Feng T, Liu WG, Luo XG, et al. Prevalence of wearing-off and dyskinesia among the patients with Parkinson's disease on levodopa therapy: a multi-center registry survey in mainland China. Transl Neurodegener. 2014;3:26.

12. Fahn S, Oakes D, Shoulson I, Kieburtz K, Rudolph A, Lang A, et al. The Parkinson study group. Levodopa and the progression of Parkinson's disease. N Engl J Med. 2004;351:2498-508.

13. Hauser RA, Panisset M, Abbruzzese G, Mancione L, Dronamraju N, Kakarieka A. FIRST-STEP study group. Double-blind trial of levodopa/carbidopa/ entacapone versus levodopa/carbidopa in early Parkinson's disease. Mov Disord. 2009;24:541-50.

14. Stocchi F, Antonini A, Barone P, Tinazzi M, Zappia M, Onofrj M, et al. DEEP study group. Early DEtection of wEaring off in Parkinson disease: the DEEP study. Parkinsonism Relat Disord. 2014;20:204-11.

15. Jenner P. Treatment of the later stages of Parkinson's disease - pharmacological approaches now and in the future. Transl Neurodegener. 2015:4:3.

16. Rascol O, Brooks DJ, Melamed E, Oertel W, Poewe W, Stocchi F, et al. LARGO study group. Rasagiline as an adjunct to levodopa in patients with Parkinson's disease and motor fluctuations (LARGO, lasting effect in adjunct therapy with Rasagiline given once daily, study): a randomised, doubleblind, parallel-group trial. Lancet. 2005;365:947-54.

17. Parkinson Study Group. A randomized placebo-controlled trial of rasagiline in levodopa-treated patients with Parkinson disease and motor fluctuations: the PRESTO study. Arch Neurol. 2005;62:241-8.

18. Dashtipour K, Chen JJ, Kani C, Bahjri K, Ghamsary M. Clinical outcomes in patients with Parkinson's disease treated with a monoamine oxidase type-B inhibitor: a cross-sectional, cohort study. Pharmacotherapy. 2015;35:681-6.

19. Chen S, Chan P, Sun S, Chen H, Zhang B, Le W, et al. The recommendations of Chinese Parkinson's disease and movement disorder society consensus on therapeutic management of Parkinson's disease. Transl Neurodegener. 2016;5:12.

20. Youdim MBH, Gross A, Finberg JPM. Rasagiline [N-propargyl-1R(+ )-aminoindan], a selective and potent inhibitor of mitochondrial monoamine oxidase B. Br J Pharmacol. 2001;132:500-6.

21. Teva Pharmaceutical Industries Ltd. Summary of Product Characteristics (SPC): Azilect ${ }^{\oplus}$ (rasagiline). 1 September 2017.

22. Parkinson Study Group. A controlled trial of rasagiline in early Parkinson disease: the TEMPO study. Arch Neurol. 2002;59:1937-43.

23. Olanow CW, Rascol O, Hauser R, Feigin PD, Jankovic J, Lang A, et al. ADAGIO study investigators. A double-blind, delayed-start trial of rasagiline in Parkinson's disease. N Engl J Med. 2009;361:1268-78.

24. Rabey JM, Sagi I, Huberman M, Melamed E, Korczyn A, Giladi N, et al. Rasagiline study group. Rasagiline mesylate, a new MAO-B inhibitor for the treatment of Parkinson's disease: a double-blind study as adjunctive therapy to levodopa. Clin Neuropharmacol. 2000;23:324-30.

25. Zhang L, Zhang Z, Chen Y, Qin X, Zhou H, Zhang C, et al. Efficacy and safety of rasagiline as an adjunct to levodopa treatment in Chinese patients with Parkinson's disease: a randomized, double-blind, parallel-controlled, multi-Centre trial. Int J Neuropsychopharmacol. 2013;16:1529-37.

26. ClinicalTrials.gov. Rasagiline in early Parkinson's disease patients not treated with levodopa in China. NCT01556165. Available from: https://clinicaltrials. gov/ct2/show/NCT01556165. Accessed 25 June 2018

27. Cai JP, Chen WJ, Lin Y, Cai B, Wang N. Safety and efficacy of rasagiline in addition to levodopa for the treatment of idiopathic Parkinson's disease: a meta-analysis of randomised controlled trials. Eur Neurol. 2015;73:5-12.

28. Hauser RA, Abler V, Eyal E, Eliaz RE. Efficacy of rasagiline in early Parkinson's disease: a meta-analysis of data from the TEMPO and ADAGIO studies. Int J Neurosci. 2016;126:942-6.

29. Chang Y, Wang LB, Li D, Lei K, Liu SY. Efficacy of rasagiline for the treatment of Parkinson's disease: an updated meta-analysis. Ann Med. 2017;49:421-34.

30. Zhou W, LV C, Zhang Q, Zong S, Wang M. Pharmacokinetics, pharmacodynamics, and safety of rasagiline transdermal patch: a preliminary study in healthy Chinese subjects. Clin Drug Investig. 2017; [Epub ahead of print]

31. China Food and Drug Administration. Provisions for Drug Registration (SFDA Order No. 28). Available from: http://www.sfdachina.com/info/64-1. htm. Accessed 25 June 2018

32. World Medical Association (WMA). Declaration of Helsinki: Ethical principles for medical research involving human subjects: Available from: www.wma. net/wp-content/uploads/2016/11/DoH-Oct2013-JAMA.pdf. Accessed 25 June 2018.

33. Hauser RA, Friedlander J, Zesiewicz TA, Adler CH, Seeberger LC, O'Brien CF, et al. A home diary to assess functional status in patients with Parkinson's 
disease with motor fluctuations and dyskinesia. Clin Neuropharmacol. 2000; 23:75-81.

34. Guy W. ECDEU assessment manual for psychopharmacology Revised edition. Rockville: National Institute of Mental Health; 1976. p. p217-22.

35. Fahn S, Elton R. UPDRS development committee. The unified Parkinson's disease rating scale. In: Fahn S, Marsden CD, Calne DB, Goldstein M, editors. Recent developments in Parkinson's disease. Volume 2. Florham Park: Macmillan health care information; 1987. p. p153-63.

36. The EuroQol Group. EuroQol: a new facility for the measurement of healthrelated quality of life. Health Policy. 1990;16:199-208.

37. Peto V, Jenkinson C, Fitzpatrick R, Greenhall R. The development and validation of a short measure of functioning and well-being for individuals with Parkinson's disease. Qual Life Res. 1995;4:241-8.

38. Stocchi F, Fossati C, Torti M. Rasagiline for the treatment of Parkinson's disease: an update. Expert Opin Pharmacother. 2015;16:2231-41.

39. de la Fuente-Fernández R, Stoessl AJ. The placebo effect in Parkinson's disease. Trends Neurosci. 2002;25:302-6.

40. Oken BS. Placebo effects: clinical aspects and neurobiology. Brain. 2008;131: 2812-23.

41. Schmidt L, Braun EK, Wager TD, Shohamy D. Mind matters: placebo enhances reward learning in Parkinson's disease. Nat Neurosci. 2014;17:1793-7.

42. Ashar YK, Chang LJ, Wager TD. Brain mechanisms of the placebo effect: an affective appraisal account. Annu Rev Clin Psychol. 2017;13:73-98.

43. Im JH, Lee JK, Chung SJ, Jeon BS, Cho JH, Lee MS, et al. Efficacy and safety of entacapone in the patients with Parkinson's disease experiencing wearing-off phenomenon: multicenter randomized placebo-controlled double blind study. J Korean Neurol Assoc. 2005;23:206-14.

44. Hauser RA, Stocchi F, Rascol O, Huyck SB, Capece R, Ho TW, et al. Preladenant as an adjunctive therapy with levodopa in Parkinson disease: two randomized clinical trials and lessons learned. JAMA Neurol. 2015 72(12):1491-500

45. Zhang ZX, Chen H, Chen SD, Shao M, Sun SG, Qu QM, et al. Chinese culture permeation in the treatment of Parkinson disease: a cross-sectional study in four regions of China. BMC Res Notes. 2014;7:65.

46. Chen X, Zhao Q, Jiang J, Liu J, Hu P. Pharmacokinetics of rasagiline in healthy adult Chinese volunteers with various genotypes: a single-center, open-label, multiple-dose study. Clin Drug Investig. 2016:36:369-76.

\section{Ready to submit your research? Choose BMC and benefit from:}

- fast, convenient online submission

- thorough peer review by experienced researchers in your field

- rapid publication on acceptance

- support for research data, including large and complex data types

- gold Open Access which fosters wider collaboration and increased citations - maximum visibility for your research: over $100 \mathrm{M}$ website views per year

At BMC, research is always in progress.

Learn more biomedcentral.com/submissions 